\title{
Software support for precise analysis of the lung ultrasound comet-tail artifact (B-line)
}

ALAN ŠUSTIĆ ( $\square)$ Department of Anesthesiology and ICU University Hospital Centre Rijeka T. Strižića 3, Rijeka 51000, Croatia E-mail: alan.sustic@medri.uniri.hr

\section{ALAN SUSTIC · ALEN PROTIC • JURICA JURANIC • ZORAN MATIC}

One of the most significant features of lung ultrasound (LUS) is comet tail artifacts, also called B-lines or "lung rockets". The causes of such phenomena are fluid-thickened interlobular septa which are the consequence of increased extravascular lung water (EVLW) or pulmonary edema. The B-lines are defined as a discrete, echogenic, vertical, laser-like signal with a narrow origin in the near field of the image. (1) Functionally, they are a sign of dysfunction of the alveolar-capillary membrane and they can be understood as the ultrasonic equivalent of Kerley B lines on X-ray. The sum of the number of B-lines correlates with the amount of EVLW in the lung and in each scanning site (generally 8 sites, 4 on the right and 4 on the left hemithorax) and may be counted from zero to ten. In addition to the estimated EVLW, detection of B-lines by LUS is a very useful tool for the diagnosis of cardiogenic versus noncardiogenic pulmonary edema and for excluding pneumothorax. However, from a clinical point of view, there are several limitations in the routine detection of B-lines. In critically ill obese patients B-lines are often more difficult to detect and vaguely visible, and sometimes it is tricky to distinguish a B-line from the so-called $\mathrm{Z}$ line. When $B$-lines are numerous, it is not easy to clearly enumerate them, especially when they tend to be confluent and in such cases the distance between B-lines cannot be precisely measured. Also, B-lines are more dynamic than static ultrasound (US) signs and they are therefore partly operator dependent signs. Mainly, for this reason, B-lines are adopted in routine clinical practice as a semiquantitative not quantitative index of EVLW accumulation. (2)

In order to maximally reduce the impact of operator experience and inter- or intra observer variability we designed software (figure 1) which calculates the number of B-lines in real time and marks them on the screen for a given time period (10 sec.). The new software was adapted for two ultrasound machines (Aloka SSD 3500 and Philips iE 33) and tested on twenty intensive care unit (ICU) patients in different stages of pulmonary edema. The degree of edema was estimated using lung computed tomography (CT) and by measuring the value of EVLW. Then, the estimated degree of edema was compared with the number of B-lines obtained by the software for each patient.

Although ultrasound examinations were performed by five examiners with different levels of experience with LUS, we found excellent correlation between the degree of lung edema and the number of B-lines displayed by the software $(r=0,85 ; p<0,01)$.

In conclusion, we found that this new support software is very useful for more precisely estimating the number of B-lines, regardless of the experience of the examiner. However, accurate assessment of the usability of the new software in routine ICU practice requires further clinical trials.

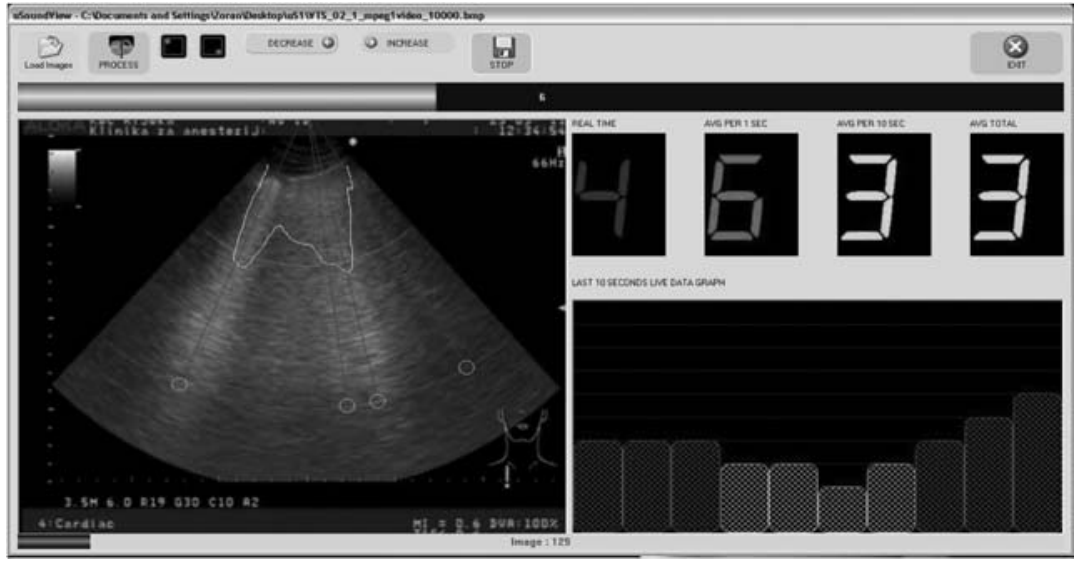

Figure 1. Software display of the number and intensity of B lines.

\section{REFERENCES}

1. Picano E, Frassi F, Agricola E, Gligorova S, Gargani L, Mottola G. Ultrasound lung comets: a clinically useful sign of extravascular lung water. J Am Soc Echocardiography 2006;19:356-63.

2. Baldi G, Gargani L, Abramo A, D'Errico L, Caramella D, Picano E, et al. Lung water assessment by lung ultrasonography in intensive care; a pilot study. Intensive Care Med 2013;39:74-84. 